\title{
An investigation on endoscopic laryngopharyngeal surgery and related outcomes
}

\author{
Yohei Kawasaki ${ }^{1}$, Yasufumi Omori ${ }^{2}$, Hidekazu Saito ${ }^{1}$, Shinsuke Suzuki ${ }^{1}$, Tamotsu Matsuhashi ${ }^{3}$, Takechiyo Yamada $^{1}$ \\ ${ }^{1}$ Department of Otorhinolaryngology and Head-and-Neck Surgery, Akita University, Graduate School of Medicine, Akita, Japan \\ 2Department of Molecular and Tumour Pathology, Akita University, Graduate School of Medicine, Akita, Japan \\ ${ }^{3}$ Department of Gastroenterology, Akita University, Graduate School of Medicine, Akita, Japan
}

Videosurgery Miniinv 2018; 13 (3): 394-400

DOI: https://doi.org/10.5114/wiitm.2018.76956

\begin{abstract}
Introduction: Robotic surgery is used in Europe and the US for oropharyngeal/hypopharyngeal cancers. Although robots can successfully perform procedures that are too delicate for surgeons and quickly learn accurate techniques, robotic surgery is not still authorized for the craniocervical region in Japan. In Japan, endoscopic laryngopharyngeal surgery (ELPS) is widely performed. Because oropharyngeal/hypopharyngeal cancer can be resected at an early stage, we have contributed to an improvement in the survival rate.

Aim: To analyze clinical outcomes and risk factors of postoperative cervical lymph node metastases after ELPS.

Material and methods: Fifty-two patients with 71 superficial oropharyngeal/hypopharyngeal cancers were included. A Sato-type arcuation laryngoscope was inserted, and oropharyngeal and hypopharyngeal fields were secured. We have recently been performing head and neck surgery using only a flexible endoscope because gastroscopy and arcuation-type forceps interfere with each other.

Results: The 5-year survival rate was 95.2\%. The risk factors of lymph node metastases were examined. The depth of the tumor significantly affected lymph node metastases.

Conclusions: With a favorable 5-year survival rate and low functional impairment, ELPS is an extremely effective form of treatment. It can provide a clear field of view in the hypopharynx and has a low cost; hence, it should be further developed as a treatment method.
\end{abstract}

Key words: endoscopic surgery, narrowband imaging, oropharyngeal cancer, hypopharyngeal cancer.

\section{Introduction}

In many cases, head and neck cancer is discovered when lymph node metastases occur or the primary tumor becomes gigantic; prognosis remains poor even with the latest developments in chemotherapy and radiotherapy [1]. Furthermore, depending on the choice of the surgical method, vocal and swallowing functions can be greatly affected, resulting in a marked decline in the patient's quality of life (QOL). Among patients who undergo radiation ther- apy, nearly half develop dysphagia and there is no significant difference in the mortality rate because of aspiration pneumonia and primary disease [2].

Recent advances in endoscopic technology have made it possible to detect cancers at a very early stage, which was previously impossible [3-8]. However, because some locations in the hypopharynx cannot be observed while the patient is in an usual position, cancer can sometimes only be detected when the patient assumes the position according to the modified Killian's method (Photo 1) [9].

\section{Address for correspondence}

Yasufumi Omori, Department of Molecular and Tumour Pathology, Akita University, Graduate School of Medicine, 1-1-1 Hondo, Akita 010-8543, Japan, phone: +81 18884 6059, e-mail: yasu@med.akita-u.ac.jp 

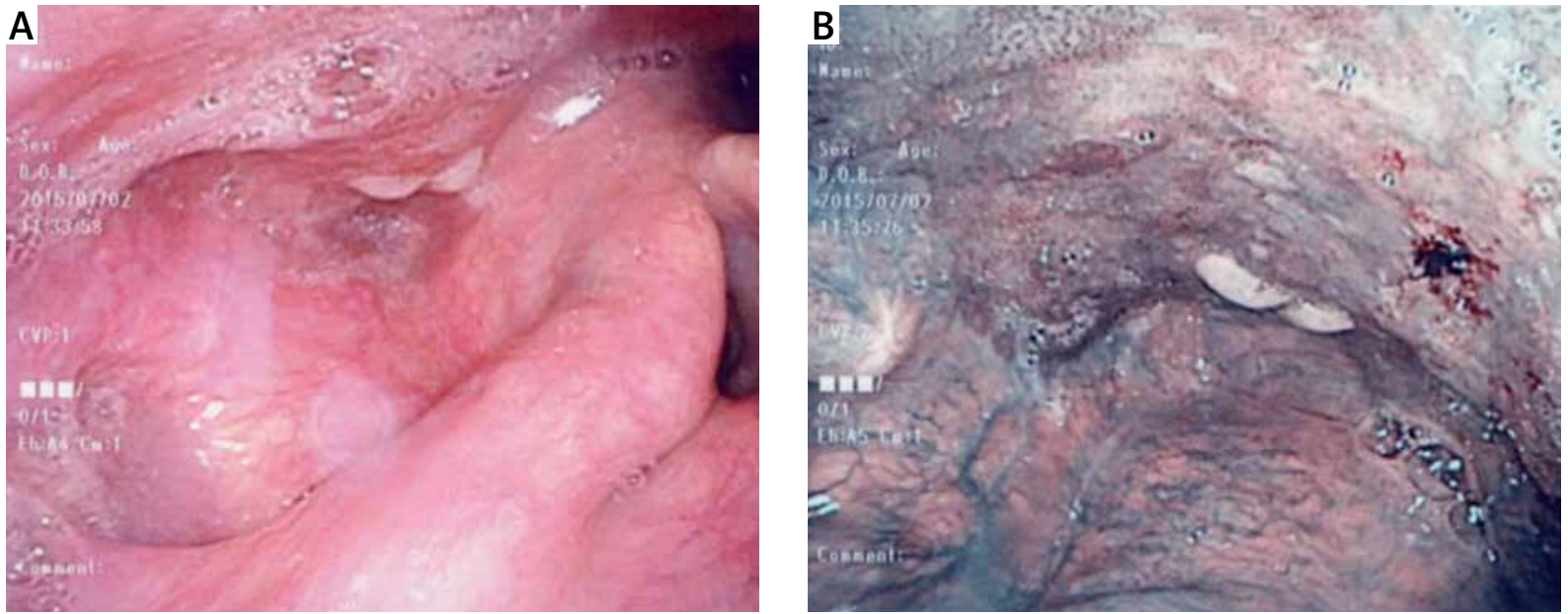

Photo 1. Differences in the appearance of hypopharyngeal cancer when viewed using normal light and narrowband imaging (NBI). A - Hypopharyngeal cancer observed using normal light. B - A brownish area is observed using NBI. Hypopharyngeal cancer is detected clearly

Narrowband imaging (NBI) has been developed for use in gastroenterological medicine; hence, early-stage head and neck cancer was often detected by gastroenterologists. Initially, gastroenterologists performed oral tumor resection for head and neck cancer using the same surgical methods employed for removing esophageal cancer, such as endoscopic mucosal resection and endoscopic submucosal dissection. However, in 2007, our institution began to use endoscopic laryngopharyngeal surgery (ELPS) in collaboration with the Department of Gastroenterology to treat issues concerning airway obstruction, delayed lymph node metastases, and local recurrence.

Robotic surgery is used in Europe and the US for oropharyngeal and hypopharyngeal cancer. Although robots can perform surgery that is too delicate for humans and quickly learn surgical techniques more accurately [10-13], robotic surgery for the craniocervical region is yet to be approved in Japan. Hence, surgery must be manually performed using a robotic arm. In other words, it is thought that this situation is the primary reason for the widespread use of ELPS.

Here we investigated the tumor site, risk factors of delayed lymph node metastases, and survival rates among 71 cases in which lesions were operated on in our institution.

\section{Aim}

In this study, we aimed to analyze clinical outcomes and risk factors of postoperative cervical lymph node metastases after performing ELPS.

\section{Material and methods}

Methods and instruments used were similar to those employed by Tateya et al. (Photo 2) [5]. We expanded the larynx using a curved laryngoscope blade (Nagashima Medical Instruments Co., Ltd, Tokyo, Japan) and secured the field of vision before inserting an endoscope with a movable tip (Olympus Medical Systems Corp., Tokyo, Japan). The area was stained with $0.75 \%$ Lugol's solution. When the stain at the tumor site had faded to a pink color, we marked a 3-mm margin and injected a saline solution containing epinephrine while using an electrosurgical knife (Olympus Medical Systems) and curved forceps (Nagashima Medical Instruments) to remove the tumor using enbloc resection. Any bleeding at the site was stopped using curved tip bipolar forceps (Nagashima Medical Instruments), and the larynx was checked to ensure that there was no swelling before completing the procedure (Photo 3). Using an endoscope with a movable tip meant that the surgery could be performed without the aid of a gastroenterologist; surgery was solely performed by otorhinolaryngologists (Photo 4). The procedure is indicated for $\mathrm{T} 1$ and $\mathrm{T} 2$ cases and some T3 cases of oropharyngeal, hypopharyngeal, and epiglottis carcinomas, but the final decision was largely dependent on the mobility of the tumor.

We obtained informed consent from all patients before their enrollment in this study. This study was reviewed and approved by the Research Ethics Committee of Akita University Hospital and was conducted in accordance with the Declaration of Helsinki. 


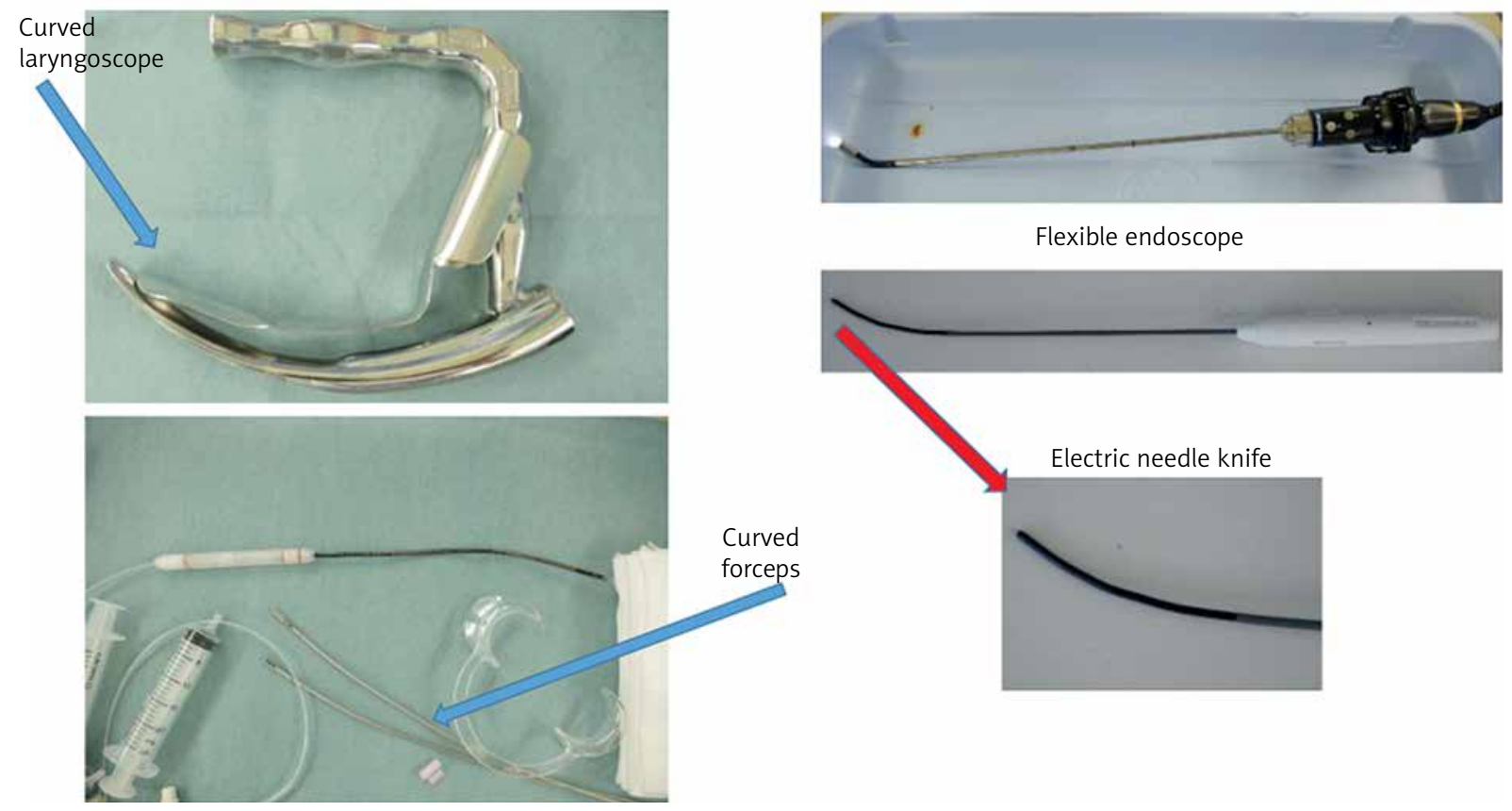

Photo 2. Surgical instruments: Sato's curved laryngoscope, rigid endoscope with a movable tip, curved forceps, and electronic needle knife

\section{Results}

Patient characteristics and tumor locations are shown in Tables I and II, respectively. An overwhelming majority of patients were male, and the most common tumor site was the pyriform sinus of the hypopharynx. Risk factors of postoperative lymph node metastases were investigated and are depicted in Table III. Delayed lymph node metastases were observed in the subepithelial layer (the tumor invades the subepithelial layer) and muscularis propria (the tumor invades the muscularis propria) [14]; there were torose lesions according to macroscopic staging classification (Table IV) [15]. The 5-year survival rate for patients with early-stage cancer who were treated with resection by ELPS was compared with that for patients who were treated using chemoradiotherapy (CRT) and analyzed using the Kaplan-Meier method (Figure 1). Although it is impossible to confirm whether the whole lesion was removed using ELPS in previous cases and because there are insufficient data on the use of ELPS to confidently draw any conclusions, as the procedure has not been commonly used for long, there was a significant difference in the survival rate. This suggests that, in many cases, patients were able to avoid radiation therapy and maintain their QOL.

\section{Discussion}

We investigated cases of patients treated with ELPS at our institution from 2007. With a 5-year disease-specific survival rate of $95.4 \%$, the results were extremely favorable. It is possible to avoid severe dysphagia and decreased salivation caused by radiation therapy as well as death due to aspiration pneumonia caused by side effects of ELPS. Furthermore, it is possible to avoid the loss of vocal function resulting from major surgery requiring external dissection. Recent advances in endoscopic technology have made it possible for otorhinolaryngologists to detect cancers at a very early stage, which is extremely important for cases of head and neck cancer. To date, there have been only two reported cases wherein tracheotomy has been performed. In 1 case, the procedure was performed in advance to avoid postoperative problems due to the poor overall condition of the patient. In the other case, laryngeal edema developed because of lengthy surgery time when the procedure was performed under the guidance of a gastroenterologist. In this study, ELPS was performed, when appropriate, in Tis, T1, and T2, and in relatively safe areas of $\mathrm{T} 3$, such as the posterior wall of the oropharynx. Cases with preoperative lymph node metastases were excluded. No postoper- 

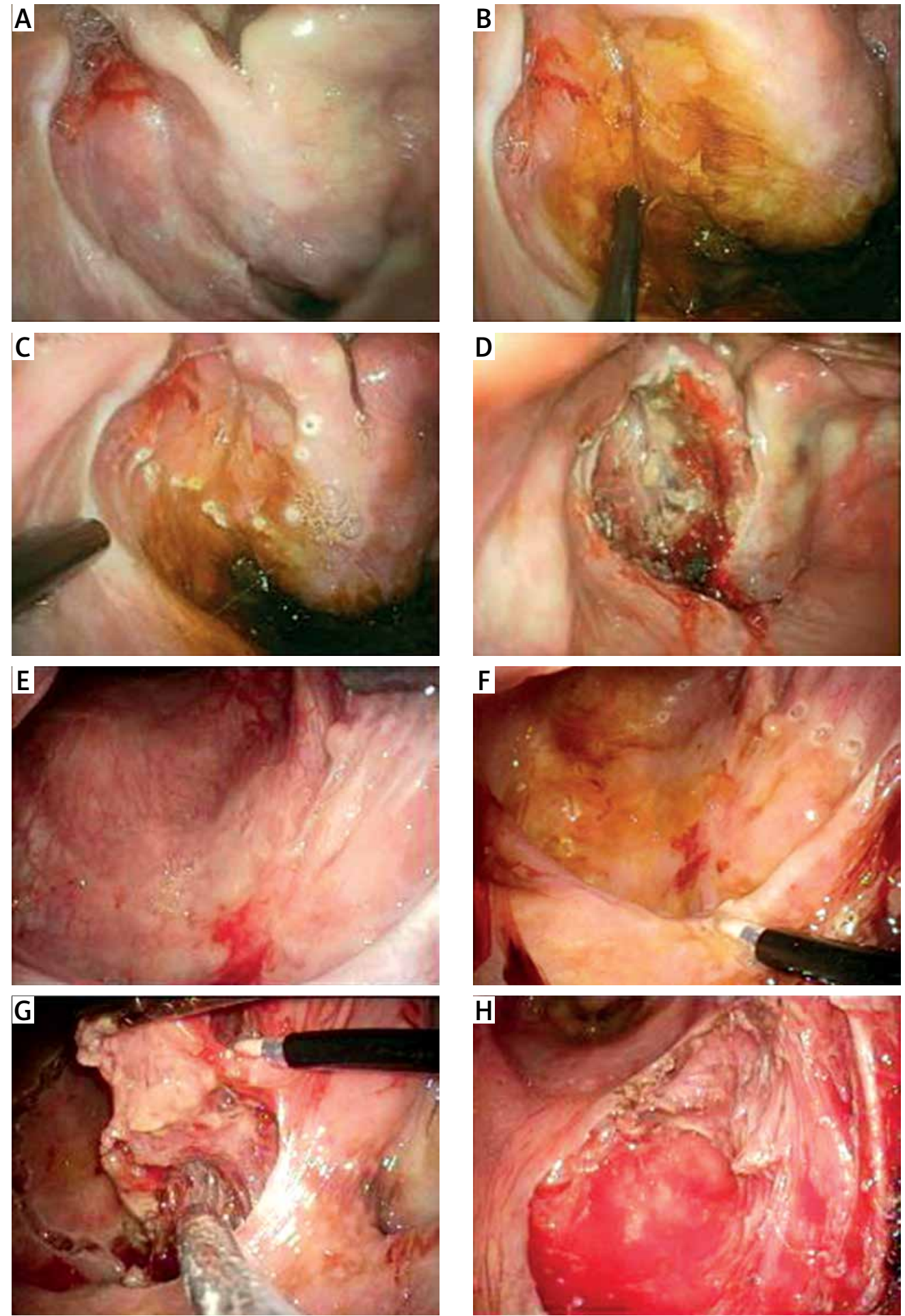

Photo 3. Surgical procedure. Cancer of the pyriform sinus of the hypopharynx: A - insert the endoscope, B - check the area stained using Lugol's solution, $\mathbf{C}$ - mark the area to be resected, D - resect the tumor with an electrosurgical knife while pulling on the forceps. Oropharyngeal cancer. Similar to the procedure performed for the hypopharynx, check the areas stained using Lugol's solution (E-G). In this case, the tumor has spread from the lateral wall to the posterior wall. $\mathbf{H}$-After marking the area to be resected, resect the tumor with an electrosurgical knife while pulling on the forceps 

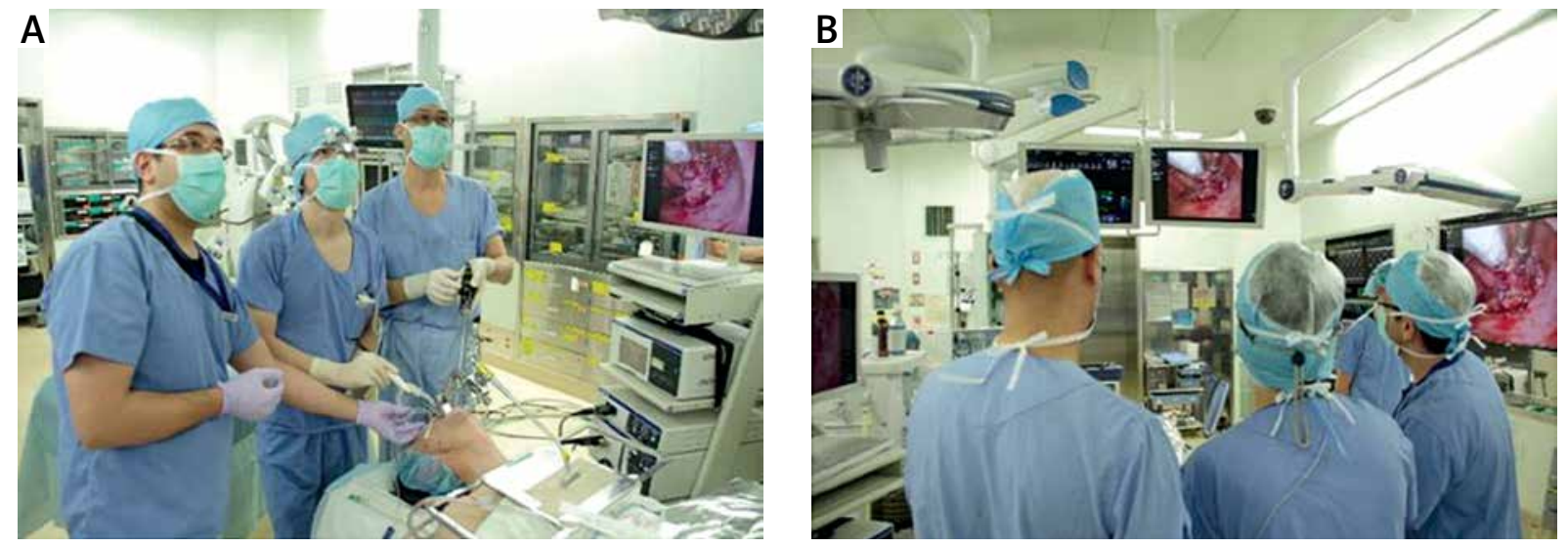

Photo 4. The person in the middle is the surgeon. The person on the right is the operator of the endoscope with the movable tip, who also injects the saline solution containing epinephrine. They are both otorhinolaryngologists. A - anterior view, $\mathbf{B}$ - posterior view

Table I. Patient characteristics

\begin{tabular}{|lc|}
\hline Parameter & Results \\
\hline Number of cases & 52 \\
\hline Number of lesions resected & 71 \\
\hline Age & 69.9 years \\
\hline Sex & Male 47; female 5 \\
\hline
\end{tabular}

ative bleeding or stenosis and severe complications, such as pharyngeal fistula, have been experienced to date, unlike those experienced in other facilities.

Postoperative lymph node metastases can be a problem, and caution is required for protuberant tumors and tumors that reach the submucosal layers; thus, routine follow-up is important. At our institution, Honda et al. performed sentinel lymph node biopsy for early-stage tongue cancer, which was extremely effective [16]. Therefore, investigations are underway to determine whether this technique can be applied to oropharyngeal and hypopharyngeal cancers.

Table III. Risk factors of postoperative lymph node metastasis (pathological classification)

\begin{tabular}{|lcc|}
\hline Variable & $\begin{array}{c}\text { Lymph node } \\
\text { metastasis (+) }\end{array}$ & $\begin{array}{c}\text { Lymph node } \\
\text { metastasis (-) }\end{array}$ \\
\hline EP & $0(0 \%)$ & $41(100 \%)$ \\
\hline SEP + MP & $4(13.3 \%)$ & $26(86.7 \%)$ \\
\hline
\end{tabular}

Notes: depth of tumor invasion (pathological classification); Fisher's exact test; $p=0.01$. EP-carcinoma in situ, SEP-tumor invades subepithelial layer, $M P$ - tumor invades muscularis propria.
Table II. Lesion characteristics

\begin{tabular}{|lcc|}
\hline Origin & $\begin{array}{c}\text { Number } \\
\text { of lesions }\end{array}$ & Subsite \\
\hline Hypopharynx & 51 & Piriform sinus: 35 \\
\cline { 3 - 3 } & & Post-cricoid: 2 \\
\cline { 3 - 3 } & & Posterior wall: 11 \\
\hline Oropharynx & 17 & Lateral wall: 3 \\
\cline { 2 - 3 } & & Posterior wall: 14 \\
\hline Larynx & 3 & Supraglottic: 3 \\
\hline Total & 71 & \\
\hline
\end{tabular}

With the increase in the aged population, there has been an increase in the number of patients requesting endoscopic surgery, even for advanced cancer with lymph node metastases. Although resection of the primary tumor using ELPS is performed after neck dissection, this is a new procedure at our institution, and more data are required to properly ascertain

Table IV. Risk factors of postoperative lymph node metastasis (macroscopic classification)

\begin{tabular}{|lcc|}
\hline Variable & $\begin{array}{c}\text { Lymph node } \\
\text { metastasis (+) }\end{array}$ & $\begin{array}{c}\text { Lymph node } \\
\text { metastasis (-) }\end{array}$ \\
\hline I + IIa & $4(11.1 \%)$ & $32(88.9 \%)$ \\
\hline Ilb + Ilc & $0(0 \%)$ & $35(100 \%)$ \\
\hline
\end{tabular}

Notes: Subtype of tumor (macroscopic classification); Fisher's exact test; $p=$ 0.04 . I - superficial and protruding type (> $1 \mathrm{~mm}$ in depth), Ila - slightly elevated type (<1 mm), Ilb-flat type, I/c - slightly depressed type $(<0.5 \mathrm{~mm})$. 


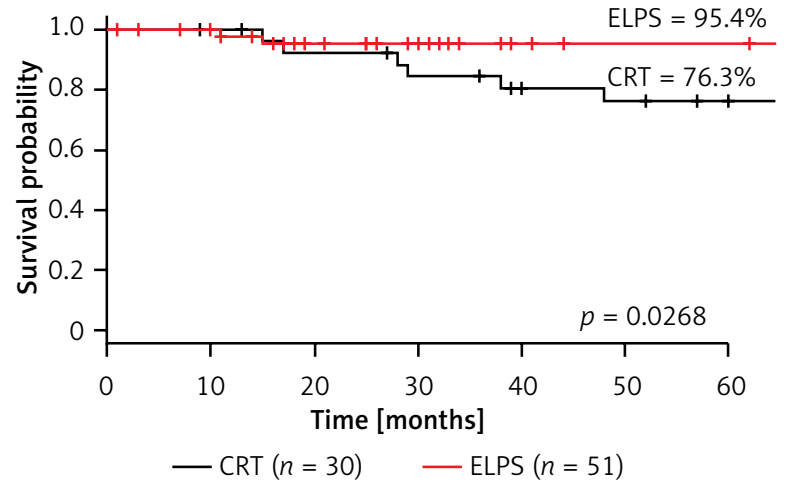

Figure 1. Kaplan-Meier analysis of the 5-year disease-specific survival for stage I + II oropharyngeal and hypopharyngeal cancers comparing CRT and ELPS. The ELPS group has a higher survival rate than the CRT group. Although there was some correlation with the degree of tumor progression, the usefulness of ELPS was observed. The 5-year disease-specific survival after ELPS for oropharyngeal and hypopharyngeal cancer is $95.4 \%$ (95\% confidence interval: $0.729-0.982$ )

the benefits. Moreover, there has been an increasing tendency to perform salvage surgery because it is now possible to detect relapses after CRT at an early stage. Although, at present, complete resection is performed, it is extremely difficult as the extent of tumor development is unclear because of hardening of the mucous membranes. Careful observation of subsequent progress is also required. Although endoscopic surgery is now performed by otorhinolaryngologists without assistance, the advantages of working with a gastroenterologist must not be forgotten because they are indispensable when it comes to cases where areas such as the cervical esophagus are involved. Therefore, depending on the site of the lesion, it is necessary to work together as a team.

\section{Conclusions}

The ELPS is an extremely useful and minimally invasive form of surgery; hence, further development is expected in future. The procedure is characterized by a short duration, reduced blood loss, and low invasiveness, which collectively make it an optimal choice for elderly patients who are more likely to have other comorbidities. It has a 5-year disease-specific survival rate of $95.4 \%$ and is more effective than robotic surgery, particularly in terms of visibility for hypopharyngeal cancer. It is also attractive because of its low cost. ELPS has the advantage of being able to easily provide good visibility of the pyriform sinus of the hypopharynx and esophageal orifice, and although the procedure is complicated to learn, we believe that its use will quickly become widespread throughout Japan.

\section{Acknowledgments}

This work is supported, in part, by JSPS KAKENHI Grant Number 15k20177.

\section{Conflict of interest}

The authors declare no conflict of interest.

\section{References}

1. Nakashima T, Yasumatsu R, Asai K, et al. Single-cycle induction chemotherapy for resectable advanced hypopharyngeal cancer. Int J Clin Oncol 2017; 22: 442-7.

2. Lindblom U, Nilsson P, Gärskog O, et al. Aspiration as a late complication after accelerated versus conventional radiotherapy in patients with head and neck cancer. Acta Otolaryngol 2016; 136: 304-11.

3. Tateya I, Shiotani A, Satou Y, et al. Transoral surgery for laryngo-pharyngeal cancer - the paradigm shift of the head and cancer treatment. Auris Nasus Larynx 2016; 43: 21-32.

4. Tateya I, Morita S, Muto M, et al. Magnifying endoscope with $\mathrm{NBI}$ to predict the depth of invasion in laryngo-pharyngreal cancer. Laryngoscope 2015; 125: 1124-9.

5. Tateya I, Muto M, Morita S, et al. Endoscopic laryngo-pharyngeal surgery for superficial laryngo-pharyngeal cancer. Surg Endosc 2016; 30: 323-9.

6. Tateya I, Ishikawa S, Morita S, et al. Magnifying endoscope with narrow band imaging to determine the surgical margin in transoral robotic surgery for superficial oropharyngeal cancer. Case Rep Otolaryngol 2014; 2014: 604-737.

7. Muto M, Nakane M, Katada C, et al. Squamous cell carcinoma in situ at oropharyngeal and hypopharyngeal mucosal sites. Cancer 2014; 101: 1375-81.

8. Watanabe A, Tsujie H, Taniguchi M, et al. Laryngoscopic detection of pharyngeal carcinoma in situ with narrowband imaging. Laryngoscope 2006; 116: 650-4.

9. Sakai A, Osaki K, Sugimoto R, et al. A new technique to expose the hypopharyngeal space: the modified Killian's method. Auris Nasus Larynx 2014; 21: 207-10.

10. Hockstein NG, Nolan JP, O'Malley BW Jr, Woo YJ. Robotic microlaryngeal surgery: a technical robot and an airway mannequin. Laryngoscope 2005; 115: 780-5.

11. Weinstein GS, O'Mallery BW Jr, Hockstein NG. Transoral robotic surgery: suraglottic laryngectomy in a canine model. Laryngoscope 2005; 115: 1315-9.

12. O'Malley BW Jr, Weinstein GS, Snyder W, et al. Transoral robotic surgery for tongue neoplasms. Laryngoscope 2006; 116: 1465-72. 
13. Weinstein GS, O'Malley BW Jr, Magnuson JS, et al. Transoral robotic surgery: a multicenter study to assess feasibility, safety, and surgical margins. Laryngoscope 2012; 122: 1701-7.

14. Japan Esophageal Society. Japanese classification of esophageal cancer, 10th edition: part 1. Esophagus 2009; 6: 1-25.

15. Japan Society for Head and Neck Cancer. General rules for clinical studies on head and neck cancer. Kanahara Publishing Group. Tokyo, Japan 2012; 58-65.

16. Honda K, Ishiyama K, Suzuki S, et al. Sentinel lymph node biopsy using computed tomographic lymphography in patients with early tongue cancer. Acta Otolaryngol 2015; 135: 136-40.

Received: 29.05.2018, accepted: 15.06.2018. 